\title{
One-year experience with the platelet glycoprotein Ilb/Illa antagonist tirofiban and heparin during cardiopulmonary bypass in patients with heparin-induced thrombocytopenia type II
}

\author{
Andreas Koster, MD, ${ }^{a}$ Oliver Meyer, MD, ${ }^{\mathrm{b}}$ Thomas Fischer, MD, ${ }^{\mathrm{a}}$ Marian Kukucka, ${ }^{\mathrm{a}}$ Thomas Krabatsch, MD, ${ }^{\mathrm{c}}$ \\ Matthias Bauer, MD, ${ }^{c}$ Herman Kuppe, MD, and Roland Hetzer, MD, ${ }^{\mathrm{c}}$ Berlin, Germany
}

$\mathrm{H}$ eparins are the standard anticoagulants in clinical medicine. However, in $1 \%$ to $3 \%$ of all patients this medication is complicated by heparin-induced thrombocytopenia type II (HIT II). This immunologic reaction causes platelet activation and is often associated with thromboses or embolism. Patients with HIT II should not be exposed to heparins. This poses a problem, particularly in cardiac surgery involving cardiopulmonary bypass (CPB), because no safe alternatives to heparin are available. The prolonged biologic elimination and unavailability of an antidote for current alternative anticoagulants, such as danaparoid or r-hirudin, may result in a persistent anticoagulant effect and severe hemorrhage.

Poetzsch, Klovekorn, and Madlener ${ }^{1}$ recently proposed that surgery be delayed until the HIT II antibodies become undetectable in laboratory assays and that unfractionated heparins then be used for anticoagulation during CPB. In those patients, after the short period of "contamination" with unfractionated heparins during CPB, HIT II antibodies remained untraceable. However, this study involved only 10 patients and lacks detail with regard to the duration of CPB. Additionally, in patients with cardiovascular disease, the operation often cannot be delayed until the antibodies are cleared, a period of approximately 40 days. ${ }^{2}$ Therefore, this strategy is applicable only for selected patients.

$\mathrm{We}^{3}$ recently introduced a new concept in which unfractionated heparins are used after platelet paralysis with the short-acting competitive platelet glycoprotein IIb/IIIa (GP IIb/IIIa) antagonist tirofiban for inhibition of HIT II-associated thromboembolism. No thromboses, thromboembolic complications, or postoperative hemorrhage were observed. However, this study involved only 10 patients. Here we report on our 1-year experience with the use of this strategy.

\footnotetext{
From the Department of Anaesthesiology, ${ }^{a}$ Deutsches Herzzentrum Berlin; Blood Bank, ${ }^{\mathrm{b}}$ Humboldt University, Charité, Campus Virchow, Berlin; and Department of Cardiothoracic and Vascular Surgery, ${ }^{\mathrm{c}}$ Deutsches Herzzentrum, Berlin, Germany.

Received for publication May 17, 2001; accepted for publication June 27, 2001.

Address for reprints: Andrea Koster, MD, Department of Anesthesiology, Deutsches Herzzentrum Berlin, Augustenburgerplatz 1, 13353 Berlin, Germany (E-mail: koster@dhzb.de).

J Thorac Cardiovasc Surg 2001;122:1254-5

Copyright (๑) 2001 by The American Association for Thoracic Surgery

$0022-5223 / 2001 \$ 35.00+0 \quad \mathbf{1 2 / 5 4 / 1 1 8 2 7 1}$

doi:10.1067/mtc.2001.118271
}

\section{Patients and Methods}

This protocol was used in 47 patients with HIT II undergoing cardiovascular surgery. In 4 patients, surgery was necessary because of acute HIT II-induced thromboembolism (pulmonary artery embolism $=2$, caval-atrial thrombus $=1$, mitral valve prosthesis thrombosis = 1). In 33 patients urgent surgery was necessary because of beginning cardiac decompensation. The remaining 10 patients underwent elective surgery. This included complex operations such as reoperations, combined procedures, transplantations, and major aortic surgery.

Tirofiban (Aggrastat; Merck \& Co, Inc, Frankfurt, Germany) was administered 10 minutes before the administration of unfractionated heparins (400 IU/kg). The dosage of tirofiban followed the RESTORE protocol with a bolus of $10 \mu \mathrm{g} \cdot \mathrm{kg}^{-1}$ and a continuous infusion of $0.15 \mu \mathrm{g} \cdot \mathrm{kg}^{-1} \cdot \mathrm{min}^{-1}$. The infusion was stopped 1 hour before the conclusion of CPB. Immediately after surgery, thrombosis prophylaxis with r-hirudin (Refludan; Aventis Pharma, Frankfurt, Germany) to a target activated partial thromboplastin time of 40 to 60 seconds was initiated.

\section{Results}

In 35 of the 47 patients, the actual presence of HIT II antibodies was detected in the heparin-induced platelet aggregation assay. In the remaining 12 patients, HIT II was diagnosed previously and the antibody status was not further evaluated.

No thromboses or embolic complications were observed either during heparinization or during the postoperative treatment. Transfusion of platelet concentrates was necessary in only 3 patients with severe preoperative thrombocytopenia $(<40,000 / \mu \mathrm{L})$. Transfusion requirements ranged from 0 to 8 units of packed red blood cells (mean $2 \pm 2.6$ units) and 0 to 9 units of fresh frozen plasma (mean $3.3 \pm 2.2$ units). The 24-hour postoperative blood loss ranged from 150 to $900 \mathrm{~mL}$ with a mean of $410 \pm 180 \mathrm{~mL}$. In all patients, transfusion requirements and postoperative blood loss were comparable with our institutional standards in patients with analogous preoperative clinical conditions and operations. No patient needed reexploration because of postoperative hemorrhage. Two patients died because of multiorgan failure as a result of myocardial failure. One patient needed prolonged care in the intensive care unit because of respiratory failure. All other patients were discharged from the hospital on schedule.

\section{Comment}

The effectiveness of platelet inhibition with GP IIb/IIIa antagonists for the treatment of acute coronary syndromes has been validated in large clinical trials. In vitro studies proved these agents to be 
able to inhibit HIT II-induced platelet aggregation. ${ }^{4}$ Combining these drugs with r-hirudin improved the outcome of patients having an acute HIT II reaction as compared with therapy with r-hirudin alone. ${ }^{5}$ These clinical data provide further convincing evidence that GP IIb/IIIa antagonists in vivo inhibit a central pathway of the HIT II cascade.

Our data clearly demonstrate that inhibition of the GP IIb/IIIa receptor during heparinization for $\mathrm{CPB}$, with HIT antibodies present, effectively prevents HIT II-associated complications. The pharmacokinetic profile of an immediate and short-acting GP $\mathrm{IIb} / \mathrm{III}$ a antagonist enables fast institution of CPB, close control of platelet aggregation, and reinstitution of hemostasis. Despite an overlap with r-hirudin during the period of recovery of platelet function, no bleeding complications were observed.

The protocol has been validated in a large number of patients. It represents a safe and practicable option for the treatment of these high-risk patients, even in cases of emergency and complex surgery.

We are indebted to Ms Anne Gale for her editorial assistance.

\section{References}

1. Poetzsch B, Klovekorn WP, Madlener K. Use of heparin during cardiopulmonary bypass in patients with a history of heparin-induced thrombocytopenia. $N$ Engl J Med. 2000;343:515.

2. Harenberg J, Wang L, Hoffmann U, Huhle G, Feurig M. Improved laboratory confirmation of heparin-induced thrombocytopenia type II: time course of antibodies and combination of antigen and biologic assays. Am J Clin Pathol. 2001;115:432-8.

3. Koster A, Kukucka M, Bach F, Meyer O, Fischer T, Mertzlufft F, et al. Anticoagulation during cardiopulmonary bypass in patients with heparin-induced thrombocytopenia type II and renal impairment using heparin and the platelet glycoprotein IIb/IIIa antagonist tirofiban. Anesthesiology. 2001;94:245-51.

4. Mak KH, Kottke-Marchant K, Brooks LM, Topol EJ. In vitro effect of platelet glycoprotein IIb/IIIa antagonist in blocking platelet function in plasma of patients with heparin-induced thrombocytopenia. Thromb Haemost. 1998;80:989-93.

5. Walenga JM, Jeske WP, Wallis DE, Bakhos M, Lewis BE, Leya F, et al. Clinical experience with combined treatment of thrombin inhibitors and GP IIb/IIIa inhibitors in patients with HIT. Semin Thromb Hemost. 1999;25(Suppl 1):77-81. 